\title{
Animal free applications in the development of cell-based therapies
}

\author{
Peter van Meer ${ }^{1}$, Peter Theunissen ${ }^{2}$, Tineke van den Hoorn ${ }^{2}$, Carla Herberts ${ }^{2}$, and Jan \\ Willem van der Laan ${ }^{2}$ \\ ${ }^{1}$ College ter Beoordeling van Geneesmiddelen \\ ${ }^{2}$ Medicines Evaluation Board
}

May 12, 2020

Commentary:

Animal free applications in the development of cell-based therapies

Peter van Meer, Peter Theunissen, Tineke van den Hoorn, Carla Herberts and Jan Willem van der Laan

Medicines Evaluation Board, Graadt van Roggenweg 500, 3531 AH, Utrecht, the Netherlands

Corresponding author: Dr. Jan Willem van der Laan, j.w.vd.laan@cbg-meb.nl

In 2018, the Dutch government launched a program called Transition towards Animal Free Innovation. This program is intended to increase the pace of animal-free innovation in the evaluation of safety of pharmaceuticals, food and chemicals through temporary, flexible networks and projects. The National Institute for Public Health and the Environment (RIVM) and the Medicines Evaluation Board (MEB) were asked how regulation of the pharmaceutical field could contribute to this programme. This prompted the drafting of a comprehensive plan to evaluate the need for animal studies in advanced therapy medicinal products (ATMPs). As ATMP technology matures, regulatory agencies, including ours, are seeing a dramatic increase in requests for scientific advice and applications for marketing authorisation of ATMPs [1]. However, the relevance of and need for (conventional) animal studies in the development of cell-based therapies is under discussion. [2]. The philosophy behind our research was the opportunity to pre-empt the incorporation of conventional animal studies before difficult to revise guidance was in place. The MEB plan included

(1) retrospective research of scientific advice procedures on to gain information on the number and type of animal studies performed and planned for cell-based therapies and

(2) a workshop with key stakeholders from academia, industry sponsors and regulatory authorities on ATMPs and specifically cell-based therapies to discuss the requirement for - and value of animals studies.

In case the drug is a human cell product, the conventional approach of testing pharmacology, pharmacokinetics and toxicity in animals may not be appropriate as species differences will limit the relevance of these studies. Generally, two routes are taken. Either (1) animal cells, surrogates of the human product, are tested in animals (homologous model) or (2) the clinical product consisting of human cells are often tested in immunocompromised animals (heterologous models). In the retrospective research (data not shown), preliminary evaluation of (genetically modified) autologous and allogenic cell-based products showed that when in vivo studies were conducted, this was generally in highly modified (e.g. with an immune-compromised system) animals. Products were generally considered safe, and the animal studies served primarily to demonstrate the expected hypothesis (i.e. expected pharmacology) (unpublished results). Cell-based therapy medicinal products require a tailored approach when assessing their safety profile including knowledge about biodistribution and tumorigenic potential (see the paper of Van den Hoorn in this issue [3]). While biodistribution 
animal studies are feasible and informative for the mechanism of action and the safety of cell-based therapies, the need for in vivotumorigenicity studies for these products is questionable. In vitro studies might be sufficiently informative (Sato et al, 2019 [4]).

In a workshop, relevant stakeholders were asked to use their extensive insights in current and future opportunities to discuss options for a more tailored, possibly animal free approach to develop cell-based therapies. A shared concept among all stakeholders was that animal free development is a rational consequence of hypothesis-driven drug development using the best tools, having human relevance in mind. A discussion in the first part of the workshop was aiming at identifying these hurdles and brainstorming for potential solutions. The second part of the workshop consisted of an exercise to develop an imaginary cell-based product by means of a tailored approach avoiding use of animal studies.

A first hurdle identified was the incline of sponsors to follow a conventional development plan also for cellbased therapies. However, a more tailored approach not necessarily requiring animal studies, may be more appropriate. Via evidence-based discussions with academia, sponsors and regulators at the European and global level (e.g. EMA CAT, SWP, SAWP and possibly ICH), the human relevance of and need for animal studies during development of cell-based therapies should be evaluated. This requires also participation of leading scientists and education of both users and policy makers in this field. These parties might form a 'coalition of the willing' that can drive change. This can be made tangible by applying for (more frequent) scientific advice procedures, which allows regulators and industry to be in contact at very early stages of drug development.

Also limited information in the public domain on the relevance of animal studies for development of cellbased therapies was recognized as a hurdle. Therefore, global developments of data sharing and transparency between stakeholders need to be initiated and/or further increased. This could lead to potential obviation of the need for animal studies as it is likely that more information on the relevance of animal studies for cell-based therapy development will become available. Cases where animal or clinical data led to a discontinuation of development should be shared in the same manner. This requires a change in mind-set by peer-reviewers of leading medical journals as well, since animal free experimentation by university groups meets significant pushback and peer reviewers will require in vivo studies in order to deem a study eligible for publication. This will facilitate continuous (retrospective) research into the need for animal studies and can help to further establish criteria where animal studies are not or no longer needed.

A third hurdle may be unfamiliarity with the advancing technology facilitating in vitro analysis or clinical testing possibilities. Where possible, it should be stimulated to use multiple novel animal-free technologies to allow evidence building. It is important to note that these in vitro, in silico and ex vivotechnologies should be evaluated and qualified within a context of establishment and subsequent use of a test system in order to be fit-for-purpose. To facilitate use of novel test systems, guidance may be needed to explain proper use of the tools and integration of the data. In addition, advancing technologies may allow collection of certain pharmacology, kinetic or safety data directly in patients. This can only be achieved when it is ethically allowed to treat human beings with the cell-based therapy in development. Supporting data for early first-in-man may come from literature data, in vitro studies or possibly in vivo animal data. However, incorporation of in vitro, in silico and ex vivo analyses in the non-clinical development phase and exploring options for investigating product aspects in the clinic, e.g. studying biodistribution directly in human, could result in a vast reduction of the number of animal studies required.

In the second part of the workshop, Break-out groups faced the challenge to develop a safe and efficacious imaginary cell-based therapy without using animal studies. The discussions resulted in hypothesis-driven programmes using state of the art technologies, but without departing from the basic principles of drug development. The resulting development steps are shown in Figure 1.

It remains difficult to substitute all animal studies with in vitro and in silico approaches or address certain aspects in the clinic. Demonstrating proof of concept $(\mathrm{PoC})$ - in order to show that a drug is able to modulate disease in a complex system - remains a requirement that is not easily supplemented by in vitro 
methods. To achieve reduction and refinement of animal use is still possible by merging safety and biodistribution endpoints in the (final) PoC study where scientifically justifiable. This requires careful design of the studies. Nevertheless, animal studies remain not always conducted based on scientific considerations but are sometimes performed because of strategic and procedural reasons. To paraphrase one of the participants: "While coming up with good arguments to perform animal studies for ATMPs is difficult, not doing them is even more difficult".

In conclusion, animal free development of cell-based therapies may be a viable endeavour but requires considerable attention. Key activities involve knowledge dissemination and activation of scientific networks of sponsors, medical doctors and toxicological-pathological experts ([5]) to generate global buy-in to change towards focussing on human centric approaches and hypothesis driven evidence generation. Sponsors as well as regulatory agencies should be stimulated to become involved as early as possible, which can be achieved using existing scientific platforms such as Public-Private Partnerships. Data generated should be made public in a transparent manner. Where in vitro, in silico and ex vivo test systems exist, evaluation on a fit-for-purpose basis is required. Use of novel test systems will require guidance to facilitate proper use of the tools and integration of the data.

Many opportunities require extensive investments of resources and while of benefit, are not in a stage where these can be readily exploited. A change of mindset from conventional towards more tailored, and where possible animal free, approaches might be a prime candidate for fostering global buy-in and activating sponsors. In addition, it is important to note that a one-fits-all guidance for cell-based products should not be the goal of this discussion. It can be suggested to regulatory authorities and sponsors to reflect explicitly on the possibility to develop cell-based products with a long experience (dendritic cells, T-cells, MSC) without animal testing. The discussion on the possibilities for applying $3 \mathrm{R}$ principle in development of cell-based therapies may be continued by hosting international workshops. This is considered a highly cost-effective manner to come to a global uniform opinion on approaching the development of cell-based therapies in a human centric and hypothesis manner

Acknowledgements

The MEB working group wishes to thank all the participants of the workshop for their valuable contributions, Anne Kienhuis and Arnoud de Kleine for their support in the development of this workshop, and the ministry of Agriculture, the Ministry of Health and Transition to Animal Free Innovation for the received funding

Conflict of interest

None declared.

\section{References}

1. Barkholt L,, Voltz-Girolt C, Raine J, Salmonson T, Schüssler-Lenz (2018) European regulatory experience with advanced therapy medicinal products. Nature Reviews/Drug Discovery

2. Broichhausen C, Riquelme P, Ahrens N, Wege AK, Koehl GE, Schlitt HJ, Banas B, Fändrich F, Geissler EK, Hutchonson JA (2014) In question: the scientific value of preclinical safet pharmacology and toxicology studies with cell-based therapies. Molecular Therapy-Methods\&Clinical Development 1, 14026: doi:10.1038/mtm.2014.26

3. Van den Hoorn T, Nakchedi T, de Wolf ACMT, Pasmooij AMG, Van der Laan JW, Herberts CA. Mining Scientific Advices on cellular therapeutics, insight in the nonclinical development program. (in this issue)

4. Sato Y, Bando H, Di Piazza M, Gowing G, Herberts CA, Jackman S, Leoni G, Libertini S, MacLachlan T, McBlane JW, Pereira Mouriès L, Sharpe M, Shingleton W, Surmacz-Cordle B, Yamamoto K, van der Laan JW, Tumorigenicity assessment of cell therapy products: The need for global consensus and points to consider. Cytotherapy, 2019, 21: 1095-1111; https://doi.org/10.1016/j.jcyt.2019.10.001)

5. Bradley AE, Black L. Evaluation of Stem Cell-Derived Cellular Therapy Products. Toxicological Pathology 2020, 1-6, DOI:10.1177/019262331|9897898. 


\section{Hosted file}

Figure 1 manuscript Animal free applications.docx available at https://authorea.com/ users/320931/articles/450331-animal-free-applications-in-the-development-of-cell-basedtherapies 